\title{
Interbody Fusion in Low Grade Lumbar Spondylolsithesis: Clinical Outcome Does Not Correalte with Slip Reduction and Neural Foraminal Dimension
}

\author{
Ujjwal K. Debnath, Atanu Chatterjee, Jeffrey R. McConnell, Deepak K. Jha, Tapas Chakraburtty
}

Department of Orthopaedic Surgery, Ramakrishna Mission Seva Pratisthan, Kolkata, India

\begin{abstract}
Study Design: Prospective nonrandomized study.
Purpose: To find a possible correlation between clinical outcome and extent of lumbar spondylolisthesis reduction.

Overview of Literature: There is no consensus in the literature concerning whether a beneficial effect of reduction on outcome can be expected following reduction and surgical fusion for low grade lumbar spondylolisthesis.

Methods: Forty six patients with a mean age of 37.5 years (age, 17-48 years) with isthmic spondylolisthesis underwent interbody fusion with cages with posterior instrumentation (TLIF). Clinical outcome was measured using visual analogue score (VAS) and Oswestry disability index (ODI). Foraminal dimensions and disc heights were measured in standard digital radiographs. These were analyzed at baseline and 1 year after surgery and changes were compared. Radiographic fusion was judged with computed tomography scans at 1 year.

Results: Ninety percent of the patients had good or very good clinical results with fusion and instrumentation. Baseline and one-year postoperative mean VAS score was 6.33 (range, $5-8$ ) and 0.76 (range, $0-3$ ), respectively $(p=0.004)$. Baseline and one-year postoperative, mean ODI score was 48 (range, 32-62) and 10 (range, 6-16), respectively $(p<0.001)$. A mean spondylolisthesis slip of $32.1 \%$ was reduced to $6.7 \%$ at 1 year. Average anterior disc height, posterior disc height, vertical foraminal dimension), and foraminal) diameter improved from 9.8 to $11.7 \mathrm{~mm}(p=0.005), 4.5$ to $5.8 \mathrm{~mm}(p=0.004), 11.3$ to $12.6 \mathrm{~mm}(p=0.002)$, and 18.6 to $20.0 \mathrm{~mm}(p<0.001)$, respectively. The fusion rate was $75 \%$ with TLIF. There is no significant correlation between the improvements of ODI scores and the extent of slip reduction.

Conclusions: Neural decompression and interbody fusion can significantly improve pain and disability but the clinical outcome does not correlate with radiological improvement in the neural foraminal dimension.
\end{abstract}

Keywords: Lumbar; Spondylolisthesis; Isthmic; Spinal fusion; Transforaminal lumbar interbody fusion

\section{Introduction}

Lumbar spondylolisthesis is a common spinal disorder in adults affecting approximately $5 \%$ of the general population [1-5]. Slipping of the cranial vertebra over the caudal vertebra leads to changes in the neural foramen morphol-

\footnotetext{
Received Jun 9, 2015; Revised Aug 2, 2015; Accepted Aug 14, 2015

Corresponding author: Ujjwal K. Debnath

Department of Orthopaedic and Spine, Ramakrishna Mission Seva Pratisthan, Kolkata, West Bengal, India, Pin: 700026

Tel: +91-90-5158-8823, Fax:+91-99-0307-2823, E-mail: ukdeb86@hotmail.com
} 
ogy with subsequent entrapment of the nerve root in the flattened and narrowed neural foramen. Clinical presentation is variable, ranging from mild to severe symptoms of low back pain with or without radiculopathy $[2,3,5,6]$ most commonly at L5/S1 and L4/5. Segmental spinal fusion is widely accepted as the method of choice for treatment of symptomatic cases not responding to conservative measures (activity modification, physical therapy and medication) [1,2,4,5,7-10].

All surgical techniques aim for decompression and stabilization of the involved vertebrae. A single-level posterior instrumented interbody fusion is commonly performed $[6,7]$. Posterolateral fusion (PLF), posterior lumbar interbody fusion (PLIF) and transforaminal lumbar interbody fusion (TLIF) are widely accepted fusion techniques [11]. Theoretically, reduction of the slip in spondylolisthesis may restore the original neuroforaminal morphology, which indirectly decompresses the nerve root. However, there is no consensus in the literature concerning whether a true beneficial effect of reduction on outcome can be expected.

There are arguments for and against reduction. Reduction of slip is a more extensive and expensive surgery with a higher risk of neurologic complications due to increased tension on the nerve roots during the reduction maneuver, especially in high grade listhesis [12-14]. Equally high rates of fusion and clinical improvement were described in studies with and without reduction $[1,13,15]$.

This study focuses on clinical outcome and evaluates a correlation between the radiographic extent of slip reduction and clinical improvement in patients with a singlelevel low-grade spondylolisthesis.

\section{Materials and Methods}

This prospective non randomized study involved $46 \mathrm{pa}$ tients (30 females and 16 males; mean age; 37.5 years; range; 17-48 years) with a diagnosis of grade 1 or 2 isthmic type lumbar spondylolisthesis who failed to respond to conservative therapy. Admitted to our hospital between 2009 and 2014. Surgeries were carried out by a single team consisting of the senior authors (U.K.D. and J.R.M) as lead. Inclusion criteria were a low-grade lumbar isthmic (Meyerding grade I, II), instrumented single-level interbody fusion and radiographic and clinical examination at baseline and 1-year after surgery.

Patients who reported any prior spinal surgery for spondylolisthesis or patients with an inadequate disk space for performing TLIF were excluded. This study was approved by Institutional Ethical Committee, and all patients proved informed written consent.

On admission, a questionnaire containing visual analogue score (VAS) and Oswestry disability index (ODI) queries described below was completed by the patient under supervision of a resident.

In the operation room, all patients were positioned prone. After a midline incision and complete bony exposure, transverse processes were exposed. Decompressive procedure was done thorough laminectomy, medial facetectomy and extensive foraminotomy. Then after pedicle screw fixation all patients underwent interbody fusion using interbody cages (TLIF; Capstone/Crescent, Medtronics Sofomor Danek, Fukushima, Japan) introduced through a standard transforaminal route and identical pedicle screw and rod instrumentation (M8; Medtronics). Autologous bone graft from the spinous process was used for fusion.

\section{Clinical outcome measurements}

A VAS assessment of leg pain on a $0-10$ point scale (o, no pain; 10, worst pain imaginable) was obtained preoperatively and at 1 year after surgery for both legs [16]. The highest score at baseline was considered to represent the worst affected leg; this side was used for prospective evaluation at the 1-year follow-up. The ODI was used to evaluate subjective perception of the effect of (low) back and leg pain on quality of life [17]. ODI is a standardized and validated questionnaire, scored from $0 \%$ (no disability) to $100 \%$ (total disability), and is often used to evaluate the outcome in spinal pathology.

\section{Radiographic measurements}

\section{1) Radiographs}

Consecutive radiographs were used to calculate radiographic parameters of reduction, which were subsequently correlated with the clinical outcome scores at preoperatively and 1-year postoperatively. Changes in disc heights and neuro-foraminal morphology were measured on radiographs and subsequently correlated to clinical outcome (VAS leg pain, ODI). Well-defined radiographic landmarks were used to ensure adequate reproducibility of the measurements (Fig. 1) [18]. All radiographs were 


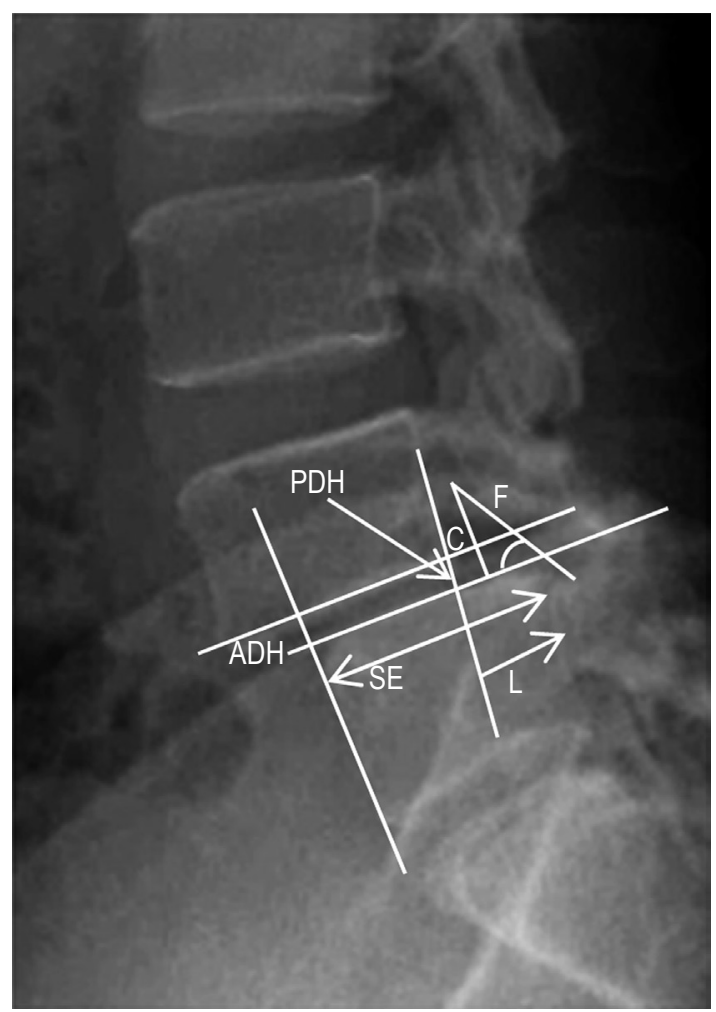

Fig. 1. Radiographic parameters measured on lateral view of radiographs of lumbar spine. $\mathrm{ADH}$, anterior disc height (distance between the intersections of the vertical line drawn from the anterior surface of the inferior vertebral body with the inferior endplate of the superior vertebra and the superior endplate of the inferior vertebra, in a $90^{\circ}$ angle with the superior endplate of the inferior vertebral body); $\mathrm{PDH}$, posterior disc height (distancebetween the intersections of the vertical line drawn from the posterior surface of the superior vertebral body with the superior endplate of the inferior vertebra and the inferior endplate of the superior vertebra, in a $90^{\circ}$ angle with the superior endplate of the inferior vertebral body); $\mathrm{C}(\mathrm{mm})$, distance from the inferior margin of the superior vertebral pedicle, to the tangent of the extended SE-line in a $90^{\circ}$ angle; a $\left({ }^{\circ}\right)$, measurement of listhesis in degrees, angle formed by the intersecting lines of $F$ and the extended SEline; Superior endplate (SE) (mm), diameter of the superior endplate of the inferior vertebral body of the affected segment; Listhesis (L), grade of listhesis measured in millimeters and percentages; Foraminal diameter (F) (mm), maximum distance measured from the inferior margin of the superior vertebral pedicle to the superior margin of the inferior vertebral pedicle.

calibrated to ensure adequate validation of the measurements due to differences in magnification between the consecutive radiographs [19]. All radiographic parameters were measured on the preoperative and postoperative lateral radiographs, and changes were recorded (Fig. 2) [20]. All measurements were performed and repeated by two blinded observers (A.C. and U.K.D.) using local PACS
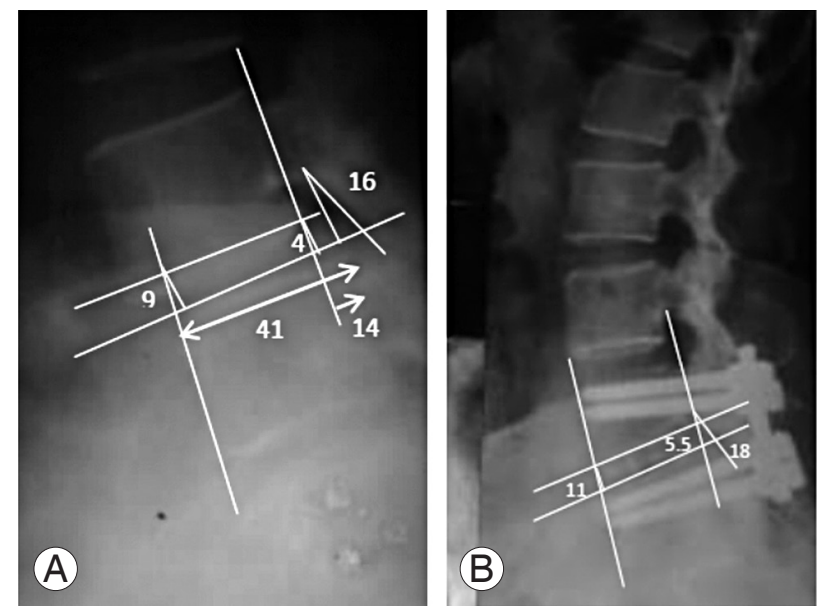

Fig. 2. (A, B) Radiographic measurements of preoperative and postoperative radiographs.

software.

2) Computed tomography (CT) scan

Pedicle screw positioning and extent of fusion was assessed in radiographs and CT scans at the 1-year followup. Fusion was assessed independently 1-year postoperatively by a radiologist and was determined according to a modification of the standardized classification system [21]. Following this classification, all patients could ultimately be classified as having fusion ("yes" or "no"). Fusion status and clinical outcome was compared and correlated between the fusion and non fusion group.

\section{Statistical analyses}

Statistical analyses were conducted using SPSS ver. 20.0 (SPSS Inc., Chicago, IL, USA). Variables were controled for normal distribution with the Kolmogorov-Smirnov test. In normally distributed data, mean, standard deviation and parametric tests (Student's $t$-test) were used to analyze differences preoperatively and postoperatively. Associations between variables were analyzed by Pearson's correlation coefficient.

Median, range and non-parametric tests (MannWhitney- $U$ and Wilcoxon-signed-rank tests) were used for analysis of non-normally distributed data. Correlation among the change in different parameters between preoperative and postoperative values are captured using Kendall's Method of correlation. Statistical significance was defined as $p<0.05$. 


\section{Results}

In the 46 patients with low grade spondylolisthesis had isthmic instability, the most commonly affected segment at L5/S1 (84\%) followed by L4/5 (16\%). All patients underwent surgery an average 12 months (range, 6-21 months) following onset of symptoms. Almost all (95\%) of the patients had predominant leg pain and radiculopathy.

\section{Clinical outcome}

Ninety percent of the patients had good or very good clinical results with TLIF and posterior pedicle screw fixa-

\section{Isthmic spondylolisthesis}

Isthmic

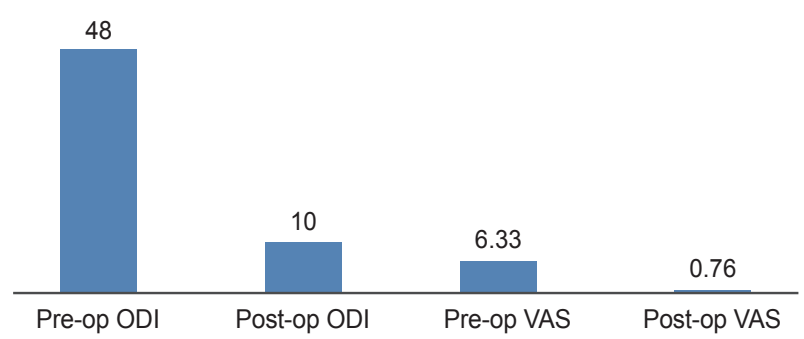

Fig. 3. Comparison of the preoperative and postoperative clinical outcome scores using ODI and VAS. ODI, Oswestry disability index; VAS, visual analogue scale. tion. VAS and ODI improved significantly at 1 year in all 46 patients. The mean VAS score at baseline and at 1-year postoperatively was 6.33 (range, $5-8$ ) and 0.76 (range, $0-3$ ), respectively $(p<0.001)$ (Fig. 3 ). The mean ODI score at baseline and 1-year postoperatively was 48 (range, 32-62) and 10 (range, 6-16), respectively $(p<0.001)$ (Table 1$)$.

\section{Radiographic outcomes}

One year following spinal fusion, the mean listhesis was $4.5 \pm 2.3 \mathrm{~mm}$, which was a significant improvement from preoperative radiograph measurements $(12.6 \pm 3.2 \mathrm{~mm})$ $(p<0.001)$ (Fig. 4). The mean percentage slip was reduced

Table 1. Data showing demographics and clinical outcome

\begin{tabular}{lcc} 
Variable & Outcome & $p$-value \\
Mean age at surgery & $37.1 \pm 9.2$ & - \\
\hline Male:female & $16: 36$ & - \\
\hline Fusion levels L5/S1:L4/5 & $44: 8$ & - \\
\hline Grade 1:grade 2 & $18: 34$ & - \\
\hline Preoperative VAS & $6.38 \pm 0.85$ & $<0.001$ \\
\hline Postoperative VAS & $0.92 \pm 0.84$ & \\
\hline Preoperative ODI & $48 \pm 8.3$ & $<0.001$ \\
\hline Postoperative ODI & $9.85 \pm 3.15$ & \\
\hline
\end{tabular}

VAS, visual analogue scale; ODI, Oswestry disability index.

\section{Isthmic spondylolisthesis}

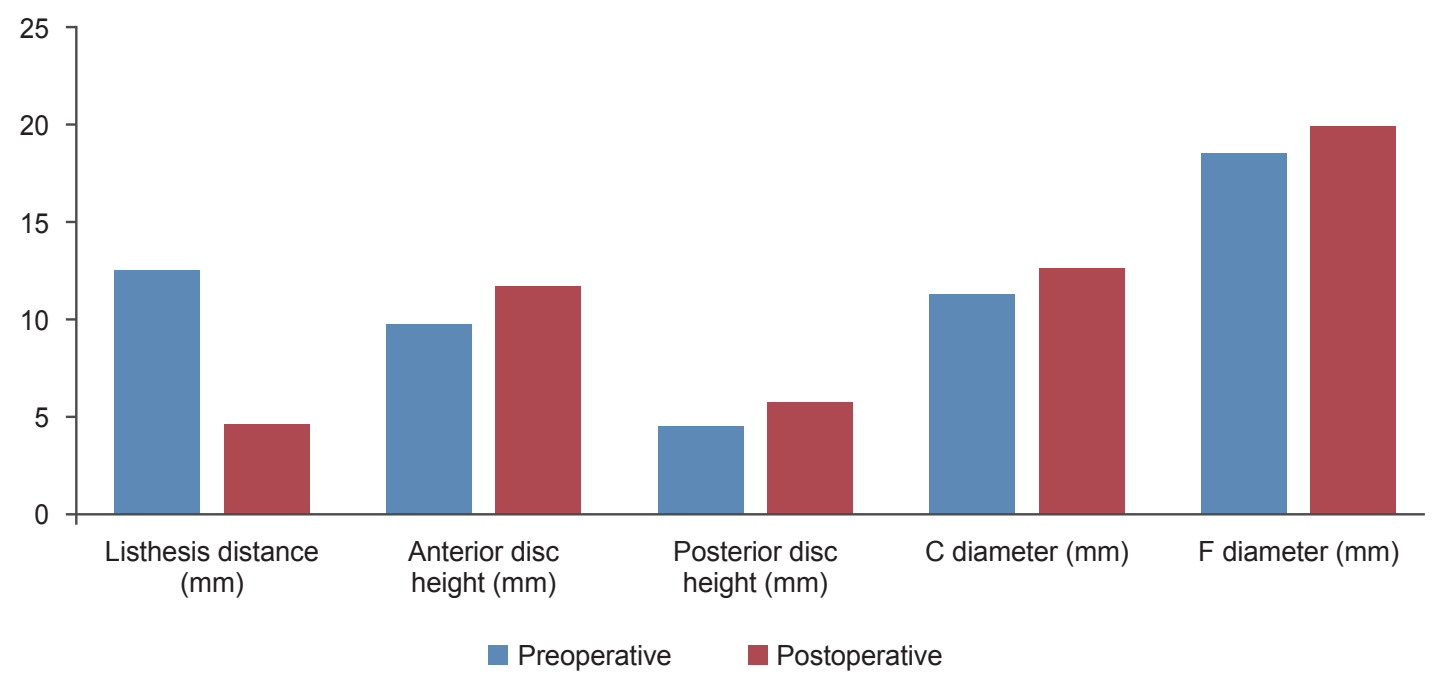

Preoperative and postoperative outcome of radiological parameters

Fig. 4. Comparison of the preoperative and postoperative radiological measurements. F, Foraminal diameter; $\mathrm{C}$, vertical distance in the foramina. 
Table 2. Correlation coefficients for clinical outcome (VAS \& ODI) at one-year postoperative and radiographic parameters

\begin{tabular}{lcc} 
& & \multicolumn{1}{c}{ Improvement at 1-year postoperative } \\
Change in radiological parameters & VAS score $(p$-value $)$ & $0.04(0.78)$ \\
\cline { 2 - 2 }$(\Delta)$ Listhesis distance $(\mathrm{mm})$ & $0.46(0.006)^{\mathrm{a})}$ & $0.5(0.7)$ \\
$(\Delta)$ Listhesis $\%$ & $0.20(0.23)$ & $0.06(0.68)$ \\
$(\Delta) \mathrm{ADH}(\mathrm{mm})$ & $0.04(0.8)$ & $0.07(0.62)$ \\
$(\Delta) \mathrm{PDH}(\mathrm{mm})$ & $0.07(0.7)$ & $0.22(0.16)$ \\
$(\Delta) \mathrm{C}$ distance $(\mathrm{mm})$ & $\left.0.44(0.01)^{\mathrm{a}}\right)$ & $0.04(0.8)$ \\
$(\Delta)$ F diameter $(\mathrm{mm})$ & $0.13(0.47)$ & $0.45(0.6)$ \\
\hline CT-scan (fusion vs. non fusion) & $0.06(0.7)$ & \\
\hline
\end{tabular}

VAS, visual analogue scale; ODI, Oswestry disability index; $\mathrm{ADH}$, anterior disc height; $\mathrm{PDH}$, posterior disc height; F, foraminal diameter; $\mathrm{C}$, vertical distance in the foramina; CT-scan, computed tomography-scan.

a) Statistically significant correlation.

significantly from $32.1 \%$ to $6.7 \%(p<0.001)$. Average anterior disc height, posterior disc height, vertical foraminal dimension ( $\mathrm{C}$ distance) and foraminal $(\mathrm{F})$ diameter improved from 9.8 to $11.7 \mathrm{~mm}$ ( $p=0.005), 4.5$ to $5.8 \mathrm{~mm}$ ( $p=0.004), 11.3$ to $12.6 \mathrm{~mm}(p=0.002)$ and 18.6 to 20.0 $\mathrm{mm}(p<0.001)$, respectively.

Thirty five (75\%) patients with CT-confirmed bony fusion at 1 year were clinically compared to analyzed the 11 'non-union' patients. Thirty six of the 46 TLIF cases showed union. The improvement from baseline in VAS scores for back and leg pain, as well as ODI scores were significant in both the fusion and non fusion groups (both $p<0.05)$, with all patients in the fusion group displaying significant improvement of VAS (back pain) than those in the non union group ( $p=0.03$ ). The VAS (leg pain) and ODI scores were comparable in both groups. There was no statistically significance correlation between solid fusion and clinical outcome; i.e., VAS (back/leg pain) or ODI scores (Table 2).

\section{Correlation}

Slip reduction measured on 1-year postoperative lateral radiographs was correlated with VAS (back and leg pain) and ODI. Pearson's correlation coefficients were significant for two radiological parameters: $(\Delta)$ listhesis $(r=0.46$, $p=0.006)$ and C distance $(r=0.44, p=0.01)$ for the improvement in the VAS scores only. All other radiological parameters had very low correlation and non-significantly different for the clinical outcomes of VAS and ODI scores. Therefore, no correlation could be established statistically between slip reduction and clinical outcome.

\section{Discussion}

Multiple options for surgical treatment of adult isthmic low grade spondylolisthesis (grade 1 and 2) are available to surgeons. Some authors recommend circumferential fusion alone with decompression and others suggest circumferential fusion with reduction of listhesis [1-3,5$7,11,14,15,22-24]$. The literature is divided regarding the surgical management with good to excellent clinical outcomes.

The surgical strategy for low grade listhesis was followed in all patients; i.e., reduction of the vertebral slip with decompression of the spinal canal and nerve roots followed by interbody fusion in the form of TLIF and postero-lateral fusion with pedicle instrumentation. Interbody fusion maintains the foraminal height and maintains structural integrity. Posterior reduction prior to the TLIF helps to restore the sagittal alignment, decompress the spinal canal effectively and maintain slip reduction $[5-7,10,11,17,24]$.

Reduction of the listhesis is commonly advocated but the relationship between reduction itself andimprovement of clinical outcome has only recently attracted clinical interest $[2,7,20]$. Comparison of PLIF with reduction versus in-situ fusion in low grade spondylolisthesis showed no influence of reduction on clinical outcome [2]. Another prospective study compared patients with and without reduction of high and low grade spondylolisthesis with only postero-lateral fusion; no difference in the outcome was apparent [7]. A study of low grade isthmic spondylo- 
listhesis could not establish any correlation of clinical outcome score improvement with the extent of radiographic reduction of spondylolisthesis in patients who underwent postero-lateral fusion [20]. In the study, there was no improvement in the neural foraminal dimensions although a wide neural decompression was achieved. Spondylolisthesis reduction was achieved through pedicle screws without extensive release of the disc and soft issues. In our series, in addition to a wide neural decompression, a discectomy and curettage of the end plate was performed for the TLIF cage insertion which maintained the reduction and improved the neural foraminal dimensions significantly.

Average anterior disc height, posterior disc height, $\mathrm{C}$ distance and $\mathrm{F}$ diameter improved significantly as compared to a similar study [20]. The maintenance of structural integrity of the interbody cages prompted the hypothesis that the clinical outcome may be related with the radiological outcome. This hypothesis failed after the results suggested that there was only moderate correlation between the VAS score and spondylolsithesis reduction distance $(r=0.46)$ and $\mathrm{C}$ diameter (vertical foraminal dimension) $(r=0.44)$. All other parameters had no correlation with VAS or ODI scores. All patients with reduction and solid fusion had good clinical outcome. This could mean that there may be moderate improvement in pain in patients who undergoes reduction of slip. Overall, the reduction of slip has no influence on the clinical outcomes scores of ODI.

There are several strengths of the study: only low grade spondylolisthesis were presented in this series and all patients had undergone the same surgery for a single level spondylolisthesis. This is the only study to date reporting a clinical and radiological correlation for Interbody fusion in low grade isthmic spondylolisthesis. The selected sample was homogeneous since all the patients were isthmic type of spondylolisthesis. The same surgeons performed the surgery. Finally, the radiographic measurements were made at only two intervals by two authors to minimize the measuring errors. Limitations of the study included being a mid-term analysis and lack of subjective outcome measures.

\section{Conclusions}

The results suggest that neural decompression and TLIF with postero-lateral instrumented fusion significantly improves pain and disability. Improvement in pain sta- tus after 1 year displayed a moderate correlation after radiographic reduction of slip and maintenance of the reduction with an interbody fusion. The overall clinical outcome was not related to the obtained radiographic reduction of the slipped vertebra.

\section{Conflict of Interest}

No potential conflict of interest relevant to this article was reported.

\section{References}

1. Jacobs WC, Vreeling A, De Kleuver M. Fusion for low-grade adult isthmic spondylolisthesis: a systematic review of the literature. Eur Spine J 2006;15:391402.

2. Audat ZM, Darwish FT, Al Barbarawi MM, et al. Surgical management of low grade isthmic spondylolisthesis; a randomized controlled study of the surgical fixation with and without reduction. Scoliosis 2011;6:14.

3. Goyal N, Wimberley DW, Hyatt A, et al. Radiographic and clinical outcomes after instrumented reduction and transforaminal lumbar interbody fusion of mid and high-grade isthmic spondylolisthesis. J Spinal Disord Tech 2009;22:321-7.

4. Kalichman L, Kim DH, Li L, Guermazi A, Berkin V, Hunter DJ. Spondylolysis and spondylolisthesis: prevalence and association with low back pain in the adult community-based population. Spine (Phila $\mathrm{Pa}$ 1976) 2009;34:199-205.

5. Floman Y, Millgram MA, Ashkenazi E, Smorgick Y, Rand N. Instrumented slip reduction and fusion for painful unstable isthmic spondylolisthesis in adults. J Spinal Disord Tech 2008;21:477-83.

6. El Masry MA, El Assuity WI, El Hawary YK, Weatherley CR. Instrumented in situ posterolateral fusion for low-grade lytic spondylolisthesis in adults. Acta Orthop Belg 2005;71:83-7.

7. Benli IT, Cicek H, Kaya A. Comparison of sagittal plane realignment and reduction with posterior instrumentation in developmental low or high dysplastic spondylolisthesis. Kobe J Med Sci 2006;52:151-69.

8. Butt MF, Dhar SA, Hakeem I, et al. In situ instrumented posterolateral fusion without decompression in symptomatic low-grade isthmic spondylolisthesis 
in adults. Int Orthop 2008;32:663-9.

9. Ekman P, Moller H, Hedlund R. The long-term effect of posterolateral fusion in adult isthmic spondylolisthesis: a randomized controlled study. Spine J 2005;5: 36-44.

10. Moller H, Hedlund R. Surgery versus conservative management in adult isthmic spondylolisthesis--a prospective randomized study: part 1 . Spine (Phila Pa 1976) 2000;25:1711-5.

11. Alijani B, Emamhadi M, Behzadnia H, et al. Posterior lumbar interbody fusion and posterolateral fusion: analogous procedures in decreasing the index of disability in patients with spondylolisthesis. Asian J Neurosurg 2015;10:51.

12. Ogilvie JW. Complications in spondylolisthesis surgery. Spine (Phila Pa 1976) 2005;30(6 Suppl):S97-101.

13. Poussa M, Remes V, Lamberg T, et al. Treatment of severe spondylolisthesis in adolescence with reduction or fusion in situ: long-term clinical, radiologic, and functional outcome. Spine (Phila Pa 1976) 2006; 31:583-90.

14. Sailhan F, Gollogly S, Roussouly P. The radiographic results and neurologic complications of instrumented reduction and fusion of high-grade spondylolisthesis without decompression of the neural elements: a retrospective review of 44 patients. Spine (Phila $\mathrm{Pa}$ 1976) 2006;31:161-9.

15. Swan J, Hurwitz E, Malek F, et al. Surgical treatment for unstable low-grade isthmic spondylolisthesis in adults: a prospective controlled study of posterior instrumented fusion compared with combined anterior-posterior fusion. Spine J 2006;6:606-14.

16. Fairbank JC, Couper J, Davies JB, O’Brien JP. The Oswestry low back pain disability questionnaire. Physiotherapy 1980;66:271-3.

17. Diedrich O, Perlick L, Schmitt O, Kraft CN. Radio- graphic characteristics on conventional radiographs after posterior lumbar interbody fusion: comparative study between radiotranslucent and radiopaque cages. J Spinal Disord 2001;14:522-32.

18. Sobottke R, Schluter-Brust K, Kaulhausen T, et al. Interspinous implants (X Stop, Wallis, Diam) for the treatment of LSS: is there a correlation between radiological parameters and clinical outcome? Eur Spine J 2009;18:1494-503.

19. American Institute of Physics; American Association of Physicists in Medicine. Standardized methods for measuring diagnostic X-ray exposures: report of task group 8 diagnostic $\mathrm{X}$-ray imaging committee. New York: American Institute of Physics; 1990.

20. Hagenmaier HS, Delawi D, Verschoor N, Oner F, van Susante JL. No correlation between slip reduction in low-grade spondylolisthesis or change in neuroforaminal morphology and clinical outcome. BMC Musculoskelet Disord 2013;14:245.

21. Andersen T, Christensen FB, Langdahl BL, et al. Fusion mass bone quality after uninstrumented spinal fusion in older patients. Eur Spine J 2010;19:2200-8.

22. Kim SS, Denis F, Lonstein JE, Winter RB. Factors affecting fusion rate in adult spondylolisthesis. Spine (Phila Pa 1976) 1990;15:979-84.

23. Carragee EJ. Single-level posterolateral arthrodesis, with or without posterior decompression, for the treatment of isthmic spondylolisthesis in adults: a prospective, randomized study. J Bone Joint Surg Am 1997;79:1175-80.

24. Spruit M, Pavlov PW, Leitao J, De Kleuver M, Anderson PG, Den Boer F. Posterior reduction and anterior lumbar interbody fusion in symptomatic low-grade adult isthmic spondylolisthesis: short-term radiological and functional outcome. Eur Spine J 2002;11:42833. 